\title{
AFFINE EXTENSIONS OF A BERNOULLI SHIFT ${ }^{1}$
}

BY

\author{
J. FELDMAN, D. J. RUDOLPH AND C. C. MOORE
}

\begin{abstract}
For any automorphism $\phi$ of a compact metric group $G$, and any $a>0$, we show the existence of a free finite measure-preserving (m.p.) action of the twisted product $\mathbf{Z} \times \boldsymbol{G}$ whose restriction to $\mathbf{Z}$ is Bernoulli with entropy $a+h(\phi), h(\phi)$ being the entropy of $\phi$ on $G$ with Haar measure.

(b) A classification is given of all free finite m.p. actions of $\mathbf{Z} \times \boldsymbol{G}$ such that the action of $\mathbf{Z}$ on the $\sigma$-algebra of invariant sets of $G$ is a Bernoulli action.

(c) The classification of (b) is extended to "quasifree" actions: those for which the isotropy subgroups are in a single conjugacy class within $G$. An existence result like that of (a) holds in this case, provided certain necessary and sufficient algebraic conditions are satisfied; similarly, an isomorphism theorem for such actions holds, under certain necessary and sufficient conditions.

(d) If $G$ is a Lie group, then all actions of $\mathbf{Z} \times \boldsymbol{\phi}$ are quasifree; if $G$ is also connected, then the second set of additional algebraic conditions alluded to in (c) is always satisfied, while the first will be satisfied only in an obvious case.

(e) Examples are given where the isomorphism theorem fails: by violation of the algebraic conditions in the quasifree case, for other reasons in the non-quasifree case.
\end{abstract}

I. Introduction. Our intention here is to complete the circle of ideas begun in [8] and [9]: to understand the structure of affine skew products over Bernoulli shifts. By an action of a group we will always mean a finite measure-preserving action.

Let $T$ be a Bernoulli shift on a space $(\Omega, \mathscr{F}, \mu)$, let $(G, \rho)$ be a compact metric group with Haar measure $\nu$, and let $\phi$ be a continuous automorphism of $G$. For any measurable map $\gamma: \Omega \rightarrow G$ we get an affine " $G, \phi$-extension" $\hat{T}$ of $T$ defined on $\Omega \times G$ by

$$
\hat{T}(\omega, g)=(T(\omega), \gamma(\omega) \phi(g)) .
$$

On this space we also have an action $\tau$ of $G$ given by

$$
\tau_{g}\left(\omega, g^{\prime}\right)=\left(\omega, g^{\prime} g^{-1}\right) \text {. }
$$

$\hat{T}$ and $\tau$ satisfy the commutation relation

$$
\tau_{\phi(g)} \circ \hat{T}=\hat{T} \circ \tau_{g} \quad \text { on } \Omega \times G,
$$

so that they combine to form an action of the twisted product group $\mathbf{Z} \times{ }^{\phi} G$. This action of $\mathbf{Z} \times{ }^{\phi} G$ we call $(\hat{T}, \tau)$. The action of $T$ on $\Omega$ can be identified

Received by the editors March 8, 1978.

AMS (MOS) subject classifications (1970). Primary 28A65, 22 D40.

${ }^{1}$ Written with partial support from NSF Grants MCS 75-05576 A01 and MCS 74-19876 A02, and from the Miller Institute for Basic Research, University of California, Berkeley. 
as the action of $\hat{T}$ on the algebra $\mathcal{Q}(\tau)$ of sets fixed by the action of $\tau$. Thus, studying $G, \phi$-extensions of $T$ is the same as studying free actions of $\mathbf{Z} \times \phi^{\phi} G$ whose $\mathbf{Z}$ part restricted to $\mathscr{Q}(\tau)$ is isomorphic to $T$.

We will say two $G$, $\phi$-extensions of $T$, call them $\hat{T}$ and $\hat{T}^{\prime}$, are $G, \phi$-isomorphic if they are isomorphic by a map $\psi$ of the form $\psi(\omega, g)=(\bar{\psi}(\omega), \alpha(\omega) \circ g)$, $\alpha: \Omega \rightarrow G$. This is equivalent to saying

$$
\bar{\psi} T^{\prime} \bar{\psi}^{-1}=T
$$

and

$$
\gamma(\omega)=\alpha(T(\omega))^{-1} \gamma^{\prime}(\psi(\omega)) \phi(\alpha(\omega))
$$

which in turn is equivalent to saying that the two $\mathbf{Z} \times^{\phi} G$ actions $(\hat{T}, \tau)$ and $\left(\hat{T}^{\prime}, \tau^{\prime}\right)$ are isomorphic.

In [9] it was shown that any two free actions of $\mathbf{Z} \times^{\phi} G$ whose $\mathbf{Z}$ subgroup actions are Bernoulli and of the same finite entropy must be isomorphic. The major unsettled question there was whether in fact such Bernoulli actions exist. This question is settled affirmatively in $\S I I$ of the present paper.

In §III, we extend the classification theory of [8] to $G, \phi$-extensions of a Bernoulli shift, showing that they can be classified up to $G, \phi$-isomorphism in a way which may be read off from the algebraic structure of the pair $(\phi, G)$.

In the subsequent sections, $\S I V, V$ and VI, we investigate whether the results of [9] and of $\S \S I$ and III can be extended to nonfree actions. Before describing these results, here is a brief analysis of such actions.

As already noted, actions of $\mathbf{Z} \times{ }^{\phi} G$ are in 1-to-1 correspondence with pairs $\bar{T}, \tau$ satisfying $\tau_{\phi(g)} \bar{T}=\bar{T} \tau_{g}$. The corresponding $\mathrm{Z} \times{ }^{\phi} G$ action we call $(\bar{T}, \tau) ; \bar{T}$ is called its $\mathbf{Z}$ part, $\tau$ its $G$ part. If $(\bar{\Omega}, \bar{F}, \bar{\mu})$ is the probability space of the action, let $(\Omega, \mathscr{F}, \mu)$ be the space of the ergodic decomposition of $\tau$. Since the $\sigma$-field of invariant sets of $\tau$ is invariant under $\bar{T}$, we get a factor $T$ of $\bar{T}$ acting on $(\Omega, \mathcal{F}, \mu)$. The fact that $G$ is compact means that the fibers of the factor map $\bar{\Omega} \rightarrow \Omega$ may be written as quotients of $G$ by closed subgroups: $H_{\omega} \backslash G$ is the fibre over $\omega$, and $\tau$ acts by the rule

$$
\tau_{g}:\left(\omega, H_{\omega} g^{\prime}\right) \mapsto\left(\omega, H_{\omega} g^{\prime} g^{-1}\right),
$$

generalizing (1.2). The isotropy subgroup of $\tau$ at $\left(\omega, H_{\omega}\right)$ is precisely $H_{\omega}$. From the relation $\tau_{\phi(g)} \bar{T}=\bar{T} \tau_{g}$ we conclude that $H_{T_{\omega}}=\phi\left(H_{\omega}\right)$. Define $\gamma(\omega)$ in $H_{\omega} \backslash G$ by $\left(\omega, H_{\omega}\right) \stackrel{T}{\mapsto}(T \omega, \gamma(\omega))$. Then $\bar{T}$ acts on $\left(\omega, H_{\omega} g\right)$ to give $(T \omega, \gamma(\omega) \phi(g))$. Conversely, given $T$ on $(\Omega, \mathscr{F}, \mu)$ satisfying $H_{T \omega}=\phi\left(H_{\omega}\right)$, and given any measurable map $\gamma$ on $\Omega$ with $\gamma(\omega) \in H_{\omega} \backslash G$, then the formula $\bar{T}\left(\omega, H_{\omega} g\right)=(T \omega, \gamma(\omega) \phi(g))$ gives a $\bar{T}$ which skew-commutes properly with $\tau$, and consequently gives a $Z \times^{\phi} G$ action. In the above discussion, there was no need to assume Bernoullicity of $T$.

Necessary and sufficient for $(\bar{T}, \tau)$ to be free are the conditions

(1) $T$ aperiodic,

(2) $\tau$ free, i.e., $H_{\omega}=\{$ id $\}$ a.e. 
Since we will mainly be concerned with the case where $T$ is a Bernoulli shift, (1) will be automatic. In this case, more generally, the only elements of $\mathbf{Z} \times{ }^{\phi} G$ which can leave anything fixed are members of $G$, so that in the nonfree case the isotropy of $(\bar{T}, \tau)$ is really just the isotropy of $\tau$.

Now, suppose we are given $\tau$ as above, and $T$ acting as a Bernoulli transformation on the $\sigma$-algebra of invariant sets of $\tau$. However, we no longer assume that $\tau$ is free. Can $\gamma$ be so chosen that $\left(\omega, H_{\omega} g\right) \mapsto(T \omega, \gamma(\omega) \phi(g))$ is a Bernoulli transformation? This we call the Bernoulli existence problem.

Suppose we have two such Bernoulli transformations: $\bar{T}$ and $\overline{\bar{T}}$. Are the actions $(\bar{T}, \tau)$ and $(\overline{\bar{T}}, \tau)$ isomorphic? (We are assuming, of course, that they are both made from the same $T$, so that they have the same entropy.) This we call the Bernoulli isomorphism problem.

Finally: can all such $\bar{T}$ (Bernoulli or not) be classified, in the manner of $\S I I I ?$ This we call the classification problem.

In $\S I V$ we deal with a special case: the quasifree case, where all the $H_{\omega}$ are conjugate in $G$. The classification problem then has an affirmative answer: the structure of §III generalizes nicely. For the Bernoulli existence problem to have an affirmative solution, there is an added necessary and sufficient algebraic condition on $G, \phi$ and the conjugacy class of subgroups involved. The condition is quite transparent, and it is easy to give counterexamples. The Bernoulli isomorphism problem also leads to an algebraic condition, somewhat more subtle.

In $\S \mathrm{V}$ it is shown that if $G$ is a Lie group then we are always in the quasifree case. Furthermore, if $G$ is also connected, then the Bernoulli isomorphism problem always has an affirmative solution; while the Bernoulli existence problem will have an affirmative solution only in a case which is already obvious from the free case: namely, when all the isotropy groups are equal to a fixed normal $\phi$-invariant subgroup.

Finally, in §VI we give three examples where the Bernoulli isomorphism theorem fails: a quasifree example with $G$ a disconnected Lie group; a quasifree example with $G$ connected but infinite dimensional; and an example with $G$ abelian but the actions not quasifree.

II. Constructing a Bernoulli $\hat{T}$. Our problem is to construct a skewing function $\gamma: \Omega \rightarrow G$ so that the $\hat{T}$ defined by (1.1) is Bernoulli. The construction rests heavily on the theory of irreducible representations of compact groups. For the reader unfamiliar with this material, an hour spent reading Chapter 2 of [4] would probably be sufficient for our task.

Let $\hat{G}=\left\{R_{1}, R_{2}, \ldots\right\}$ be a complete collection of irreducible representations for $G$, no two unitarily equivalent, with $R_{k}(g)=\left[r_{i, j}^{k}(g)\right]$ an $n(k) \times n(k)$ matrix representation. The collection of functions $\left\{\sqrt{n(k)} r_{i, j}^{k}\right\}_{i, j, k}$ form an orthonormal basis for $L^{2}(G)$.

We begin by investigating how $\phi$ acts on the irreducible representations. If $R \in \hat{G}$ we denote by $\hat{\phi}(R)$ the representation $R \circ \phi$. Then $R, \hat{\phi}(R), \hat{\phi}^{2}(R), \ldots$ form a sequence of irreducible representations. Either 
$\hat{\phi}^{n}(R)$ is unitarily equivalent to $R$ for some $n$, or all the components of all these representations are orthogonal. If the first case occurs we will say $\hat{\phi}$ is cyclic on $R$.

For any set $\Delta \subset \hat{G}$, let $\Delta^{\perp}=\{g: R(g)=I$ for all $R \in \Delta\}$. Then it is immediate that $\Delta^{\perp}$ is a closed normal subgroup of $G$. The representations in $\Delta$ give rise in a natural way to representations of $G / \Delta^{\perp}$; these will again be irreducible. Call $\Delta$ multiplicative if for each $R_{1}, R_{2} \in \mathcal{S}$ the irreducible components of the Kronecker product $R_{1} \otimes R_{2}$ are unitarily equivalent to members of $\Delta$. Say $\Delta$ is self-conjugate if for each $R \in \Delta$, with $R(g)=\left[r_{i j}(g)\right]$, the complex-conjugate $R^{*}$ given by $R^{*}(g)=\left[r_{i}^{*}(g)\right]$ is unitarily equivalent to an element of $\Delta$. The Tannaka duality theorem tells us that a necessary and sufficient condition for $\Delta$ to contain a complete set of representations of $G / \Delta^{\perp}$ is that $\Delta$ be multiplicative and self-conjugate. Also note that if $\Delta$ is $\phi$-invariant, in the sense that each $\phi(R)$ is unitarily equivalent to some element of $\Delta$, then $\Delta^{\perp}$ is $\phi$-invariant, so $\phi$ acts on $G / \Delta^{\perp}$.

Let $\Gamma_{1}$ be the set of those $R \in \hat{G}$ on which $\hat{\phi}$ is cyclic. It is easy to check that $\Gamma_{1}$ is multiplicative, self-conjugate, and $\hat{\phi}$-invariant. Thus $\Gamma_{1}^{\perp}$ is a closed $\phi$-invariant normal subgroup of $G, \Gamma_{1}$ gives rise to a complete set of irreducible representations of $G / \Gamma_{1}^{\perp}$, and $\phi$ acts on $G / \Gamma_{1}^{\perp}$. As $\hat{\phi}$ is cyclic on all elements of $\Gamma_{1}$, it is easy to see that $\phi$ on $G / \Gamma_{1}^{\perp}$ is an isometry, for a suitable metric equivalent to the given one. Note in passing that the converse is also true: if $\phi$ on $G$ is an isometry then $\Gamma_{1}^{\perp}=\{$ id $\}$.

We will see later that when $\phi$ is an isometry then $\gamma$ can be explicitly constructed to make $\hat{T}$ Bernoulli. The plan, then, is to break down $\phi$ in some way so as to somehow reduce to the isometric case. $G / \Gamma_{1}^{\perp}$ is the first step of this reduction, and we now discuss how to continue.

Let $\Delta \subset \hat{G}$ be multiplicative, self-conjugate, and $\hat{\phi}$-invariant. We want to find a multiplicative, self-conjugate, $\hat{\phi}$-invariant set $\Delta^{\prime} \supset \Delta$ for which $\phi$ acting on $\Delta^{\perp} / \Delta^{\prime}$ is an isometry. This is done (as was done for $\Delta$ empty and $\Delta^{\prime}=\Gamma_{1}$ ), by considering irreducible representations for $\Delta^{\perp}$ on which $\hat{\phi}$ is cyclic. But $\Delta^{\prime}$ is to be a subset of $\hat{G}$, so we must see how representations of $\Delta^{\perp}$ arise from those of $G$.

Certainly the restriction of any $R \in \hat{G}$ to $\Delta^{\perp}$ is a representation of $\Delta^{\perp}$, but not necessarily irreducible. There is an equivalent $S=U R U^{-1}$ where

$$
S / \Delta^{\perp}=\left[\begin{array}{cccc}
S_{1} & & & 0 \\
& S_{2} & & \\
& & \ddots & \\
0 & & & S_{l}
\end{array}\right]
$$

and each $S_{j}$ is an irreducible representation for $\Delta^{\perp}$. Further, every irreducible representation for $\Delta^{\perp}$ appears as an $S_{j}$ arising from some $R$ in this manner. As $\Delta^{\perp}$ is normal in $G$ and $R$ is irreducible, letting $g^{*}$ represent conjugation of $\Delta^{\perp}$ by $g \in G$, for each $S_{i}$ and $S_{j}$ there is a $g \in G$ with $\widehat{g^{*}}\left(S_{i}\right)$ unitarily 
equivalent to $S_{j}$; and for any $g \in G, g^{*}\left(S_{i}\right)$ must be unitarily equivalent to some $S_{j}$.

If $\hat{\phi}^{n}\left(S_{i}\right)$ is unitarily equivalent to some $S_{j}$, then for any other $S_{k}, \hat{\phi}^{n}\left(S_{k}\right)=$ $\hat{\phi}^{n}\left(\widehat{g^{*}}\left(S_{i}\right)\right)=\widehat{\phi^{n}(g)^{*}}\left(\hat{\phi}^{n}\left(S_{i}\right)\right)$ is unitarily equivalent to some $S_{t}$. Thus, up to unitary equivalence, $\hat{\phi}^{n}$ permutes $S_{1} \ldots S_{l}$, and so $\hat{\phi}^{n !}\left(S_{i}\right)$ must be unitarily equivalent to $S_{i}$ for all $i$, and $\hat{\phi}$ is cyclic on each $S_{i}$. We also have $\hat{\phi}^{n l !}\left(S / \Delta^{\perp}\right)$ unitarily equivalent to $R \mid \Delta^{\perp}$. On the other hand, if $\hat{\phi}^{n}\left(S \mid \Delta^{\perp}\right)$ is unitarily equivalent to $S \mid \Delta^{\perp}$, then each $\hat{\phi}^{n}\left(S_{i}\right)$ is unitarily equivalent to some $S_{j}$. Thus the irreducible representations for $\Delta^{\perp}$ on which $\hat{\phi}$ is cyclic are precisely those which come from $R$ 's with $\hat{\phi}$ cyclic on $R \mid \Delta^{\perp}$.

If $\hat{\phi}$ acts cyclically on the irreducible representations $S_{j}$ of $\Delta^{\perp}$, i.e., $\hat{\phi}^{n}\left(S_{j}\right)$ is unitarily equivalent to $S_{j}$, then $\hat{\phi}^{n}\left(S \mid \Delta^{\perp}\right)$ is unitarily equivalent to $R \mid \Delta^{\perp}$. Thus the irreducible representations for $\Delta^{\perp}$ on which $\hat{\phi}$ is cyclic are precisely those which come from $R$ 's with $\hat{\phi}$ cyclic on $R \mid \Delta^{\perp}$.

Now define $\Delta^{\prime}$ to be $\left\{R \in \hat{G} \mid \hat{\phi}\right.$ is cyclic on $\left.R \mid \Delta^{\perp}\right\}$.

As with $\Gamma_{1}^{\perp}, \Delta^{\prime \perp}$ is a closed $\phi$-invariant normal subgroup of $G, \Delta^{\prime \perp} \subseteq \Delta^{\perp}$ and $\phi$ acts on $\Delta^{\perp} / \Delta^{\prime \perp}$ as an isometry.

Now for any ordinal $\alpha$, define inductively $\Gamma_{\alpha}$ by setting $\Delta=\cup_{\alpha^{\prime}<\alpha^{\prime}} \Gamma_{\alpha^{\prime}}$, and then $\Gamma_{\alpha}=\Delta^{\prime}$ in the previous construction. This procedure will terminate at some countable $\bar{\alpha}$, as $\hat{G}$ is countable.

This gives a decreasing chain of subgroups $\Gamma_{\alpha}^{\perp}$ and no nontrivial irreducible representation of $\Gamma_{\bar{\alpha}}^{\perp}$ is moved cyclically by $\phi$.

We now state what the function $\gamma$ will be. Write the entropy $h(T)$ as $\Sigma_{\alpha<\bar{\alpha}} a_{\alpha}$ with $a_{\alpha}>0$, and let $(T, \Omega)=\Pi_{\alpha<\bar{\alpha}}\left(T_{\alpha}, Y_{\alpha}\right)$ where each $T_{\alpha}$ is Bernoulli and $h\left(T_{\alpha}\right)=a_{\alpha}$. Let $\mathscr{P}_{\alpha}=\left\{P_{\alpha, i}\right\}$ be a countably infinite independent generator for $T_{\alpha}$.

Define $\gamma_{\alpha}: Y_{\alpha} \rightarrow G$ by $\gamma_{\alpha}(y)=g_{\alpha, i}, y \in P_{\alpha, i}$, where $\left\{g_{\alpha, i} \Gamma_{\alpha}^{\perp}\right\}$ is dense in $\left(\cup_{\alpha^{\prime}<\alpha} \Gamma_{\alpha^{\prime}}\right)^{\perp} / \Gamma_{\alpha}^{\perp}$ and $\gamma_{\alpha}=$ id for all but $\varepsilon_{\alpha}$ of $Y_{\alpha}$. Here the $\varepsilon_{\alpha}$ are chosen $>0$, and subject to the condition that $\Sigma_{\alpha<\bar{\alpha}} \varepsilon_{\alpha}<\infty$. Set

$$
\gamma(\omega)=\prod_{\alpha<\bar{\alpha}} \gamma_{\alpha}\left(y_{\alpha}(\omega)\right) \text {. }
$$

This is well defined, as $\Sigma \varepsilon_{\alpha}<\infty$.

We will now show that the $T$ defined by $\gamma$ is Bernoulli by showing inductively for every $\alpha<\bar{\alpha}$ that

$$
\hat{T}_{\alpha}\left(\omega, g \Gamma_{\alpha}^{\perp}\right)=\left(T(\omega), \gamma(\omega) \phi(g) \Gamma_{\alpha}^{\perp}\right)
$$

is Bernoulli, and then applying the main result of Lind [6] to get from $\hat{T}_{\bar{\alpha}}$ to $\hat{T}$.

First take a limit ordinal $\alpha$, and suppose for $\alpha^{\prime}<\alpha$ that $\hat{T}_{\alpha^{\prime}}$ is Bernoulli. We want to show that $\hat{T}_{\alpha}$ is Bernoulli. But if $\alpha^{\prime \prime}<\alpha^{\prime}$ then the map $\left(\omega, g \Gamma_{a^{\prime \prime}}^{+}\right) \mapsto$ $\left(\omega, g \Gamma_{\alpha^{\prime \prime}}^{\perp}\right)$ makes $\hat{T}_{\alpha^{\prime \prime}}$ a factor of $\hat{T}_{\alpha^{\prime}}$ Thus, the $\hat{T}_{\alpha^{\prime}}, \alpha^{\prime}<\alpha$, are factors of $\hat{T}_{\alpha}$ in a consistent way, and the fact that $\Gamma_{\alpha}^{\perp}=\bigcap_{\alpha^{\prime}<\alpha} \Gamma_{\alpha^{\prime}}^{\perp}$ guarantees that the inverse images of the $\sigma$-algebras of the $\hat{T}_{\alpha^{\prime}}, \alpha^{\prime}<\alpha$, generate the $\sigma$-algebras of $\hat{T}_{\alpha}$. Since each of the $\hat{T}_{\alpha^{\prime}}$ is Bernoulli for $\alpha^{\prime}<\alpha$, it follows from Ornstein's theorem on monotone limits of Bernoulli transformations that $\hat{T}_{\alpha}$ is also Bernoulli. 
Next take a successor ordinal, $\alpha=\alpha^{\prime}+1$, and suppose $\hat{T}_{\alpha^{\prime}}$ is Bernoulli. We want to show that $\hat{T}_{\alpha}$ is Bernoulli. Set $\bar{G}=G / \Gamma_{\alpha}^{\perp}, \bar{H}=\Gamma_{\alpha^{\prime}}^{\perp} / \Gamma_{\alpha}^{\perp}$, and $\bar{\gamma}(\omega)=$ $\gamma(\omega) \Gamma_{\alpha}^{\perp}$. Write $\Omega=Y_{\alpha} \times \bar{\Omega}$, where

$$
(\bar{T}, \bar{\Omega})=\prod_{\substack{\beta<\bar{\alpha} \\ \beta \neq \alpha}}\left(T_{\beta}, Y_{\beta}\right),
$$

and split $\bar{\gamma}(\omega)$ into $\gamma_{\alpha}(y) \gamma^{\prime}(\bar{\omega})$. Then $\hat{T}$ may be written as

$$
(y, \bar{\omega}, \bar{g}) \mapsto\left(T_{\alpha}(y), \bar{T}(\bar{\omega}), \gamma_{\alpha}(y) \gamma^{\prime}(\omega) \phi(\bar{g})\right) .
$$

Write $\bar{G}$ as $\bar{G} / \bar{H} \times \bar{H}$ by using the map $(\bar{g} \bar{H}, \bar{h}) \mapsto \sigma(\bar{g} \bar{H}) \bar{h}$, with inverse $\overline{\mathbf{g}} \mapsto\left(\overline{\mathrm{g}} \bar{H}, \sigma(\overline{\mathrm{g}} \bar{H})^{-1} \bar{g}\right)$, where $\sigma$ is a measurable cross-section: $\bar{G} / \bar{H} \uparrow \bar{G}$. Then $\hat{T}_{\beta}$ becomes isomorphic to the transformation

$$
(y, \bar{\omega}, \bar{g} \bar{H}, \bar{h}) \mapsto\left(T_{\alpha}(y), \bar{T}(\bar{\omega}), \alpha^{\prime}(\bar{\omega}) \phi(\bar{g} \bar{H}), \bar{k}\right),
$$

where $\bar{k}=\sigma\left(\gamma^{\prime}(\bar{\omega}) \phi(\bar{g} \bar{H})\right)^{-1} \gamma_{\alpha}(y) \gamma^{\prime}(\bar{\omega}) \phi(\sigma(\bar{g} \bar{H}) \bar{h})$. Set

$$
\begin{aligned}
& \delta_{1}(\bar{\omega}, \bar{g} \bar{H})=\sigma\left(\gamma^{\prime}(\bar{\omega}) \phi(\bar{g} \bar{H})\right)^{-1} \gamma^{\prime}(\bar{\omega}) \phi(\sigma(\bar{g} \bar{H}))^{-1}, \\
& \delta_{2}(\bar{\omega}, \bar{g} \bar{H})=\phi(\sigma(\bar{g} \bar{H})) \gamma^{\prime}(\bar{\omega})^{-1} .
\end{aligned}
$$

Then $\delta_{1}$ takes its values in $\bar{H}$ and $\delta_{2}$ in $\bar{G}$, and our transformation becomes

$$
\begin{aligned}
(y, \bar{\omega}, \bar{g} \bar{H}, \bar{h}) & \mapsto\left(T_{\alpha}(y), \bar{T}(\bar{\omega}), \gamma^{\prime}(\bar{\omega}) \phi(\bar{g} \bar{H}),\right. \\
& \left.\delta_{1}(\bar{\omega}, \bar{g} \bar{H}) \delta_{2}(\bar{\omega}, \bar{g} \bar{H}) \gamma_{\alpha}(\bar{g}) \delta_{2}(\bar{\omega}, \bar{g} \bar{H})^{-1} \phi(\bar{h})\right) .
\end{aligned}
$$

where

$$
\delta_{1}(\bar{\omega}, g H)=\beta\left(g^{\prime}(\bar{\omega}) \phi(g h)^{-1}\right) \gamma^{\prime}(\bar{\omega}) \phi\left(\beta(H)^{-1}\right),
$$

so $\delta_{1}$ takes $\bar{\Omega} \times G / H$ to $H$, and

$$
\delta_{2}(\bar{\omega}, g H)=\phi(\beta(g H)) \gamma^{\prime}(\bar{\omega})^{-1},
$$

so $\delta_{2}$ takes $\bar{\Omega} \times G / H$ to $G$.

In (2.1) the second and third coordinates give a factor isomorphic to $\hat{T}^{\prime}$, hence a Bernoulli factor. We will show in the following proposition that this factor splits off with a Bernoulli complement, by using the ideas of Thouvenot ([10], [11]).

Proposition 2.1. Let $S$ acting on $X$ be an m.p. transformation, $(T, Y) a$ Bernoulli shift, $G$ a compact metric group, $\phi$ an automorphism of $G$. Suppose $H$ is a closed $\phi$-invariant normal subgroup of $G$ on which $\phi$ acts isometrically. Let $\gamma: Y \rightarrow H$ be measurable w.r.t. an independent generator $\mathscr{P}=\left\{P_{i}\right\}$ of $T$, and $\gamma(Y)$ dense in $H$. Let $\delta_{1}: X \rightarrow H, \delta_{2}: X \rightarrow G$ be any measurable functions. Let $\bar{T}$ from $X \times Y \times H$ to itself be given by

$$
\bar{T}(x, y, h)=\left(S(x), T(y), \delta_{1}(x) \delta_{2}(x) \gamma(y) \delta_{2}(x)^{-1} \phi(h)\right) .
$$

For any partition 2 of $H$ into open sets with boundary of zero measure, $(\bar{T}, \mathcal{P} \times \mathcal{Q})$ is relatively $V . W . B$. (see [12]) with respect to the algebra given by the first coordinate. 
Proof. We will verify something which will imply the result for any such 2. Fix a point $x \in X$ and let $\mu_{x}$ be the fiber measure on $x \times Y \times H$. This is, of course, the product measure on $Y \times H$. Now also fix an atom $f \in$ $\bigvee_{i=-1}^{-\infty} T^{-i}(\mathcal{P})$ and an element $h_{0} \in H$, and let $\bar{f}=x \times f \times h_{0}$. A point of $\bar{f}$ is specified by a future name from the $(T, \mathcal{P})$-process, and the fiber measure $\mu_{\bar{f}}$ on $\bar{f}$ is the independent measure on these names.

We would be done if we could show that for a.e. $f \times h_{0}$,

$$
\bar{d}_{N}\left(\bigvee_{i=0}^{N} \bar{T}^{-i}(\mathcal{P} \times \mathcal{2}) / x \times Y \times H, \bigvee_{i=0}^{N} \bar{T}^{-i}(\mathcal{P} \times \mathcal{Q}) / \bar{f}\right) \rightarrow 0 \text { as } N \rightarrow \infty
$$

since every past atom of $\mathscr{P} \times \mathcal{Q}$ is a union of such $x \times f \times h_{0}$.

Let $\bar{h}(x, y, h)=h$, the third coordinate. To get (2.2) we must certainly show that

$$
\lim _{N \rightarrow \infty}\left(\operatorname{dist}\left(\bar{h}\left(\bar{T}^{-i}\right) / \bar{f}\right)\right)=\text { Haar measure }
$$

on $H$. Conversely, we show that it will suffice to prove (2.3). From (2.3), for any $\varepsilon$, once $N$ is large enough, we have, in the notation of [9],

$$
\left\|\operatorname{dist}\left(\bar{h}\left(\bar{T}^{-N}\right) / \bar{f}\right), \operatorname{dist}\left(\bar{h}\left(\bar{T}^{-N}\right) / x \times Y_{2} \times H\right)\right\|<\varepsilon^{2}
$$

Let $p$ be an atom of $\bigvee_{i=N}^{\infty} T^{-1}(\mathcal{P})$. As $\mathcal{P}$ is an independent generator, independent of $(S, X)$, and as $\gamma$ is $\mathscr{P}$ measurable,

$$
\operatorname{dist}\left(\bar{h}\left(\bar{T}^{-N}\left(x \times f \cap p \times h_{0}\right)\right)\right)=\operatorname{dist}\left(\bar{h}\left(\bar{T}^{-N}(\bar{f})\right)\right) .
$$

Thus let $\Pi_{p}$ be a map: $x \times p \times H \rightarrow x \times f \cap p \times h_{p}$, measure preserving on fiber measures, so that

$$
\int_{x \times f \cap p \times h_{0}} \rho\left(\bar{h}\left(\Pi_{p}\left(\bar{T}^{-N}\right)\right), \bar{h}\left(\bar{T}^{-N}\right)\right) d \mu_{\left(x \times f \cap p \times h_{0}\right)}<\varepsilon,
$$

$\rho$ being the $\phi$-invariant metric on $H$.

But now, since $\phi$ is an isometry on $H$, for all $n>N$,

$$
\int_{x \times f \cap p \times h_{0}} \rho\left(\bar{h}\left(\Pi_{p}\left(\bar{T}^{-n}\right)\right), \bar{h}\left(\bar{T}^{-n}\right)\right) d \mu_{\left(x \times f \cap p \times h_{0}\right)}<\varepsilon .
$$

Letting $\Pi=\int_{p} \Pi_{p} d \mu_{p}$, and using this for a possible match as in [9], we get the estimate

$$
\bar{d}_{M}\left(\bigvee_{i=1}^{M} \bar{T}^{-i}(\mathscr{P} \times \bar{h}) / x \times f \times h_{0}, \bigvee_{i=1}^{M} \bar{T}^{-i}(\mathcal{P} \times \bar{h}) / x \times Y \times H\right)<2 \varepsilon
$$

for $M>N / \varepsilon\left(\bar{d}_{M}\right.$ in the sense of [9]).

\footnotetext{
${ }^{2}$ Remark. The topology on probability distributions on $G$ given by the metric $\|\cdot, \cdot\|$ is really just the restriction of the weak * topology on signed measures as the dual of $C(G)$. That the weak topology is weaker, is almost obvious. That it is also stronger, we leave as an amusing exercise for the reader; the only proof we know uses the Marriage Lemma.
} 
It is an easy argument that $\bar{h}$ in (2.4) can be replaced by a partition 2 of open sets with boundary of zero measure.

What remains is to prove (2.3). This is where representation theory enters, as this limit can be verified by evaluating it on irreducible representations. Fix $x \times f \times Q_{0}$, and let $\tau_{N}=\operatorname{dist}\left(\bar{h}\left(\bar{T}^{-1}\right) / x \times f \times h_{0}\right)$. This is a probability distribution on $H$. Let $\bar{R}$ be a nontrivial irreducible representation for $H, R$ an irreducible representation for $G$ which gives rise to $\bar{R}$. The following lemma will complete our argument.

Lemma 2.2. Using notation as above and in Proposition 2.1, $R d \tau_{n} \stackrel{n}{\rightarrow}[0]$.

Proof. Now $\tau_{0}$ is a point mass at $h_{0}$, so $\int R d \tau_{n}=R\left(h_{0}\right)$. Furthermore if $\pi_{h}$ means the point mass at $h$, and $\tau_{n}=\sum_{i} a_{i} \pi_{h_{i}}$, then

$$
\tau_{n+1}=\sum_{i, j} \mu\left(P_{j}\right) a_{i} \pi\left(\delta_{1}(x) \delta_{2}(x) h_{j} \delta_{2}(x)^{-1} \phi\left(h_{i}\right)\right)
$$

where $\gamma / P_{j} \equiv h_{j}$. Now

$$
\int R d \tau_{n+1}=\left[\sum \mu\left(P_{j}\right) R\left(\delta_{1}(x)\right) R\left(\delta_{2}(x)\right) R\left(h_{j}\right) R^{-1}\left(\delta_{2}(x)\right)\right] \int \hat{\phi}(R) d \tau_{n} .
$$

Let $A_{j}=\Sigma_{i} \mu\left(P_{i}\right) \hat{\phi}^{j}\left(R\left(h_{i}\right)\right)$. Thus

$$
\int R d \tau_{n}=\prod_{j-1}^{n-1}\left(B_{j, n} A_{j} \bar{B}_{j, n}\right) R\left(h_{0}\right),
$$

for some unitary matrices $B_{j, n}$ and $\bar{B}_{j, n}$.

We would like to show that the $A_{j}$ are strict contractions, i.e. for any $v \in \mathbf{C}^{n},\left|A_{j}(v)\right|<\rho_{j}|v|, \rho_{j}<1$, and furthermore $\rho_{j}$ is periodic in $j$. This with (2.5) will prove Lemma 2.2 .

$A_{j}$ is a convex combination of unitary matrices. Thus if $\left|A_{j}(v)\right|=|v|$ then for all $h_{i}, \hat{\phi} R^{j}\left(h_{i}\right)(v)=\hat{v}$ independent of $i$. So $V=\left\{v|| A_{j}(v)|=| v \mid\right\}$ is a closed subspace of $C^{n}$, invariant under all $\hat{\phi}^{j} R\left(h_{i}\right)$. As the $h_{i}$ are dense in $H, V$ is invariant under all $\hat{\phi}^{j} R(h), h \in H$, and on it all $\hat{\phi}^{j} R(h)$ are constant, hence the identity. But as $R / H$ is nontrivial, $V=\{0\}$ and $\rho_{j}=\sup _{|v|-1}\left|A_{j}(v)\right|<1$. Now $\phi / H$ is an isometry, for some $k$, so $\hat{\phi}^{k}(R / H)$ is unitarily equivalent to $R / H$, and hence $A_{j+k}$ is unitarily equivalent to $A_{j}$, and $\rho_{j+n}=\rho_{j}$.

Proposition 2.1 provides the induction step to show every $\hat{T}_{\alpha}$ is Bernoulli, hence $\hat{T}_{\bar{\alpha}}$. To begin the induction we need a simpler version of the same argument, which we omit. Now just as in (2.1) we can write $\hat{T}$ as isomorphic to a transformation $\bar{T}$, where

$$
\bar{T}\left(\omega, g \Gamma_{a}^{\perp}, h\right)=\left(T(\omega), \gamma(\omega) \phi(g) \Gamma_{a}^{\perp}, \bar{\gamma}\left(\omega, g \Gamma_{a}^{\perp}\right) \circ \phi(h)\right)
$$

for some $\bar{\gamma}: \Omega \times G / \Gamma_{\alpha}^{\perp} \rightarrow \Gamma_{\alpha}^{\perp}$.

$\Gamma_{\bar{\alpha}}$ has been so constructed that $\hat{\phi}$ on $\hat{G} / \Gamma_{\bar{\alpha}}$ is acyclic. But it is well known that if an automorphism of a compact abelian group has a dual which is acyclic on nonidentity characters, then the automorphism must be ergodic. The same simple argument which proves this fact will also work in the 
nonabelian case using irreducible representations rather than characters. Thus $\phi$ is ergodic on $\Gamma_{\bar{\alpha}}^{\perp}$. So Lind's work in [5] or [6] says that the factor given by the first two coordinates of (2.6) has a Bernoulli complement. Thus $\hat{T}_{\bar{\alpha}}$ in $\hat{T}$ has a Bernoulli complement and $\hat{T}$ is Bernoulli.

(2.7) REMARK. $\Gamma_{\bar{\alpha}}$ is the maximal $\phi$-invariant subgroup on which $\phi$ is ergodic, while the cosets of $\Gamma_{\bar{\alpha}}$ in $G$ are the atoms of the Pinsker algebra of the action of $\phi$ on $G$. Also, the restriction of $\phi$ to the Pinsker algebra is distal, as is clear from the construction of $\Gamma_{\bar{\alpha}}$, since it was explicitly exhibited as a limit of a sequence of isometric extensions of an isometry. See [3].

III. Classifying the $G$, $\phi$-extensions of a Bernoulli shift. Between them, the isomorphism theorem of [9] and §II tell us that up to $G, \phi$-isomorphism there is precisely one $G, \phi$-extension of a Bernoulli shift $T$ that is itself Bernoulli. As with isometric extensions [8] we will now show that this is just one instance of a classification of all $G, \phi$-extensions of $T$ up to $G, \phi$-isomorphism.

Proposition 3.1. If $\hat{T}$ is a $G$, $\phi$-extension of a finite entropy Bernoulli shift $T$, and $\hat{T}$ is weakly mixing, then $\hat{T}$ is Bernoulli.

Proof. Let $\Gamma_{\alpha}, \alpha<\bar{\alpha}$, be as defined in $\S I I$, and $\bar{T}_{\alpha}$ the factor of $\hat{T}$ with fibers $\left(\omega, g \Gamma_{\alpha}^{\perp}\right)$. As $\hat{T}$ is weakly mixing, all the $\hat{T}_{\alpha}^{\prime}$ s are. Now $\hat{T}_{0}=T$ is Bernoulli. Suppose for $\alpha^{\prime}<\alpha, \hat{T}_{\alpha^{\prime}}$ is Bernoulli. Then by Ornstein's monotone theorem $\hat{T}_{\alpha^{-}}$, the factor of $\hat{T}$ with fibers $\left(\omega, g\left(\cup_{\alpha^{\prime}<\alpha^{\prime}} \Gamma_{\alpha^{\prime}}\right)^{\perp}\right)$, is Bernoulli. Since $\hat{T}_{\alpha}$ is an isometric extension of $\hat{T}_{\alpha^{-}}$, and weakly mixing, it is Bernoulli, by [8]. But by Lind [6], as $\phi / \Gamma_{\alpha}^{\perp}$ is ergodic, $\hat{T}_{\bar{\alpha}}$ has a Bernoulli complement in $\hat{T}$.

Now let us see what happens when $\hat{T}$ has a Pinsker algebra $\Pi(\hat{T})$. The goal is to show that $\hat{T}$ acting on $\Pi(\hat{T})$ has a simple algebraic form, and that this action in fact characterizes $\hat{T}$ up to $G, \phi$-isomorphism. Our first step, which for $\phi=$ id in [8] was trivial, is to show that $\Pi(\hat{T})$ is $\tau$ invariant.

LEMMA 3.2. Let $\hat{T}$ be a G, $\phi$-extension of the m.p. transformation $T$ on $(\Omega, \mathcal{F}, \mu),(\hat{T}, \tau)$ the corresponding $\mathbf{Z} \times{ }^{\phi} G$ action. For any $g \in G, \tau_{g}(\Pi(\hat{T}))=$ $\Pi(\hat{T})$.

Proof. First a trivial case: Suppose the skewing function $\gamma(\omega) \equiv$ id. Then $\hat{T}=T \times \phi, \Pi(\hat{T})=\Pi(T) \times \Pi(\phi)$, and $\tau_{g}(\Pi(\hat{T}))=\Pi(T) \times \tau_{g}(\Pi(\phi))$. But a fiber of $\Pi(\phi)$ is a coset of $\Gamma_{\alpha}^{\perp}$, and these are certainly $\tau_{g}$ invariant, so $\Pi(\hat{T})$ is $\tau_{g}$ invariant.

Now for the general $\hat{T}(\omega, g)=(T(\omega), \gamma(\omega) \phi(g))$, define $T: \Omega \times G \times G \rightarrow \Omega$ $\times G \times G$ by

$$
\bar{T}\left(\omega, g_{1}, g_{2}\right)=\left(T(\omega), \gamma(\omega) \phi\left(g_{1}\right), \gamma(\omega) \phi\left(g_{2}\right)\right),
$$

a $G \times G, \phi \times \phi$-extension. The map $\hat{T}$ can be identified as either the first and second or first and third coordinates of this map. As we saw in Lemma 1 of [9], $\bar{T}$ is isomorphic to $\hat{T} \times \phi$ by the map $\psi\left(\omega, g_{1}, g_{2}\right)=\left(\left(\omega, g_{1}\right), g_{1}^{-1} g_{2}\right)$, and $\psi$ commutes with right multiplication by $g \in G$ on the third coordinate. Thus 
$\bar{\tau}_{(\mathrm{id}, g)}(\Pi(\bar{T}))=\tau_{g}(\Pi(\hat{T} \times \phi))=\Pi(\hat{T} \times \phi)=\Pi(\bar{T})$ by the trivial case. But identifying $\hat{T}$ as the first and third coordinates of $\bar{T}$, we are done.

LemMa 3.3. Suppose $T$ is a $G$, $\phi$-extension of a Bernoulli shift $T$, and $(\hat{T}, \tau)$ the $\mathbf{Z} \times{ }^{\phi} G$ action obtained. The action of $(\hat{T}, \tau)$ on $\Pi(\hat{T})$ is then isomorphic to $\left(g_{0} \circ \phi, G\right)$ acting on $H \backslash G$, where $H$ is a closed subgroup of $G$ containing $\Gamma_{a}^{\perp}$ and $g_{0} \phi(H)=H g_{0}$.

Proof. As $T$ is Bernoulli, $\Pi(\hat{T})$ must be independent of $\mathcal{Q}(\tau)$, the algebra of $\tau$ invariant sets. Thus $\tau$ restricted to $\Pi(\hat{T})$ is an ergodic action of $G$. Any such is isomorphic to right multiplication on some $H \backslash G, H$ a closed subgroup of $G$. Let $p: \Omega \times G \rightarrow H \backslash G$ be this projection of the action of $\tau$. Now for a.e. $\omega, p(\omega \times G)$ is onto $H \backslash G$, as these are the ergodic components of the action of $\tau$.

Now $(\omega \times G) \cap p^{-1}(H)$ is $\tau_{h}$ invariant, $h \in H$, hence is of the form $\omega \times \alpha_{\omega} H$, where $\alpha_{\omega}$ can be chosen measurably in $\omega$. Thus

$$
p^{-1}(H g)=\bigcup_{\omega \in \Omega}\left(\omega \times \alpha_{\omega} H g\right),
$$

and these are the atoms of $\Pi(\hat{T})$.

Define a $G, \phi$-isomorphic copy $\bar{T}$ of $\hat{T}$ by

$$
\bar{T}=\psi_{0} \hat{T} \psi_{0}^{-1}, \quad \psi_{0}(\omega, g)=\left(\omega, \alpha_{\omega}^{-1} g\right)
$$

As $\tau_{g} \psi_{0}=\psi_{0} \tau_{g},(\bar{T}, \tau)$ is an action of $\mathbf{Z} \times^{\phi} G$, and as $\Pi(\bar{T})=\psi_{0}^{-1}(\Pi(\hat{T}))$, the atoms of $\Pi(\bar{T})$ are of the form $\Omega \times H g$. Let

$$
\bar{\gamma}(\omega)=\alpha_{T(\omega)} \gamma(\omega) \phi\left(\alpha_{\omega}\right)^{-1}
$$

be the skewing function for $\bar{T}$.

Now $\bar{T}(\Omega \times H)=(\Omega \times \bar{\gamma}(\omega) \phi(H))$, which must be another atom of $\Pi(\bar{T})$, and so of the form $\Omega \times H g_{0}$, for some $g_{0}$. Thus $\bar{\gamma}(\omega)=\delta(\omega) g_{0}$, for some $\delta$ : $\Omega \rightarrow H$, and $\delta(\omega) g_{0} \phi(H)=H g_{0}$, so $\phi(H)=g_{0}^{-1} H g_{0}$.

Then $\bar{T}(\Omega \times H g)=\left(\Omega \times \delta(\omega) g_{0} \phi(H) \phi(g)\right)=\Omega \times g_{0} \phi(H g)$. Consequently $\hat{T}$ on $\Pi(\hat{T})$ is isomorphic to $g_{0} \phi$ acting on $H \backslash G$.

Now as we saw in (2.1) of $\S I I, g_{0} \phi$ acting on $G$ can be written as

$$
g_{0} \phi:\left(g \Gamma_{\alpha}^{\perp}, h\right) \mapsto\left(g_{0} \phi(g) \Gamma_{\alpha}^{\perp}, \varepsilon\left(g \Gamma_{\alpha}^{\perp}\right) \phi(h)\right)
$$

for some function $\varepsilon: G / \Gamma_{\alpha}^{\perp} \rightarrow \Gamma_{\alpha}^{\perp}$. The first term of this representation is the composition of a distal transformation and an isometry, by (2.7), hence itself is distal, hence of 0-entropy, and as we saw earlier, has a Bernoulli complement. Thus the atoms of $\Pi\left(g_{0} \phi\right)$ are the cosets of $\Gamma_{a}^{\perp}$, and as $g_{0} \phi$ acting on $H \backslash G$ is of 0-entropy, $\Gamma_{\alpha}^{\perp} \subseteq H$.

Notice that given an $H \supseteq \Gamma_{a}^{\perp}$, it is not always true that there exists a $g_{0}$ with $\phi(H)=g_{0}^{-1} H g_{0}$, but when there does, $g_{0} \phi$ acting on $H \backslash G$ has zero entropy.

Also notice that in this lemma the choice for $H$ is not unique; any conjugate $g^{-1} \mathrm{Hg}$ could also be chosen; but the conjugacy class is unique. Also for a given choice of $H, g_{0}$ is only unique up to the right coset of $H$ in $G$ 
containing it. In keeping with this, on the set of pairs $\left\{\left(H, g_{0}\right): H\right.$ a closed subgroup, $\Gamma_{\alpha}^{\perp} \subseteq H, g_{0}$ such that $\left.\phi(H)=g_{0}^{-1} H g_{0}\right\}$, one may define an equivalence $\left(H, g_{0}\right) \sim\left(H^{\prime}, g_{0}^{\prime}\right)$ if there are $g \in G, h \in H$ with $g H^{-1}=H^{\prime}$, $g\left(h g_{0}\right) g^{-1}=g_{0}^{\prime}$. Let $\mathbf{H}$ be the set of equivalence classes. We write the equivalence class of $\left(H, g_{0}\right)$ as $\left\langle H, g_{0}\right\rangle$. From the above remarks, for every $G$, $\phi$-extension $\hat{T}$ of $T$, there is a well-defined element $\mathcal{H}(T) \in \mathbf{H}$, from Lemma 3.3, and this element is a $G, \phi$-isomorphism invariant. We will now show that it is a complete invariant.

Proposition 3.4. If $\hat{T}$ and $\hat{T}^{\prime}$ are two $G$, $\phi$-extensions of a finite entropy Bernoulli shift $T$ and $\mathcal{H}(\hat{T})=\mathcal{H}\left(\hat{T}^{\prime}\right)$, then $\hat{T}$ and $\hat{T}^{\prime}$ are $G, \phi$-isomorphic.

Proof. Without loss of generality, as we saw in the proof of Lemma 3.3, we may assume that for some $\left(H, g_{0}\right) \in \mathcal{H}(\hat{T})$, we have two maps $\delta^{\prime}, \delta: \Omega \rightarrow H$ and

$$
\begin{aligned}
\hat{T}(\omega, g) & =\left(T(\omega), \delta(\omega) g_{0} \phi(g)\right), \\
\hat{T}^{\prime}(\omega, g) & =\left(T(\omega), \delta^{\prime}(\omega) g_{0} \phi(g)\right) .
\end{aligned}
$$

Let $\bar{T}=\tau_{g_{0}} \hat{T} / \Omega \times H, \bar{T}^{\prime}=\tau_{g_{0}} \hat{T}^{\prime} / \Omega \times H$. These are well defined, as

$$
\tau_{g_{0}} \hat{T}(\omega, h)=\left(T(\omega), \delta(\omega) g_{0} \phi(h) g_{0}^{-1}\right) \in \Omega \times H .
$$

Now $\Pi\left(\tau_{g_{0}} \hat{T}\right)=\Pi(\hat{T})$, as certainly $\Pi(\hat{T}) \subset \Pi\left(\tau_{g_{0}} \hat{T}\right)$ from the form of $\Pi(\hat{T})$, and as $\left(\tau_{8_{0}} \hat{T}, \tau\right)$ is a $\mathbf{Z} \times{ }^{8_{0}^{*} \phi} G$ action. $\Pi\left(\tau_{g_{0}} \hat{T}\right) \subset \Pi(\hat{T})$ by the same reasoning on this other group. (Again $g_{0}^{*}$ is conjugation by $g_{0}$.) Thus, as $\Omega \times H$ is an atom of $\Pi\left(\tau_{8_{0}} \hat{T}\right), \bar{T}$ is a $K$-automorphism, and similarly so is $\bar{T}^{\prime}$.

Now $\bar{T}$ and $\bar{T}^{\prime}$ are $H, g_{0}^{*} \phi$-extensions of $T$. They are $K$-automorphisms, hence weakly mixing, hence Bernoulli by Proposition 3.1. Then [9] tells us that they are $H, g_{0}^{*} \phi$-isomorphic. Let $\left(\bar{\psi}, \alpha_{\omega}\right)$ be the implementing $H, g_{0}^{*} \phi$-isomorphism.

Thus

$$
\delta(\omega)=\alpha_{T(\omega)}^{-1} \delta^{\prime}(\bar{\psi}(\omega)) g_{0}^{*} \phi\left(\alpha_{\omega}\right)
$$

or

$$
\delta(\omega) g_{0}=\alpha_{T(\omega)}^{-1} \delta(\bar{\psi}(\omega)) g_{0} \phi\left(\alpha_{\omega}\right),
$$

and (see (1.4)) $\left(\bar{\psi}, \alpha_{\omega}\right)$ is a $G, \phi$-isomorphism between $\hat{T}$ and $\hat{T}^{\prime}$.

To complete our classification we need to know that every $\left(H, g_{0}\right)$ actually arises. To do this, use $\S I I$ to construct $\delta(\omega)$ so that the $H, g_{0}^{*} H$-extension $\bar{T}$ of $T$ thereby defined is Bernoulli. Let $\gamma(\omega)=\delta(\omega) g_{0}$; then $\mathcal{H}(\hat{T})=\left\{\left(H, g_{0}\right)\right\}$. Thus any $G, \phi$-extension is isomorphic to such a one.

As a corollary of our classification we can now show that any $G, \phi$-extension of a Bernoulli shift is of the form Bernoulli $\times$ distal, i.e. the Pinsker algebra has a Bernoulli complement. As with Lemma 3.2, the proof is a bit of algebraic chicanery. First a lemma. 
LEMMA 3.5. Let $S$ acting on $\left(\Omega^{\prime}, \delta^{\prime}, \mu^{\prime}\right)$ be a 0 -entropy system, and $h^{\prime}: \Omega^{\prime} \rightarrow G$ be measurable. Then

$$
\hat{S}\left(\omega^{\prime}, g\right)=\left(S\left(\omega^{\prime}\right), h^{\prime}\left(\omega^{\prime}\right) \phi(g)\right)
$$

is of the form 0-entropy $\times$ Bernoulli.

Proof. Write $G=G / \Gamma_{a}^{\perp} \times \Gamma_{\alpha}^{\perp}$; and as we saw earlier $\hat{S}$ thus becomes isomorphic to

$$
\hat{S}^{\prime}\left(\omega^{\prime}, g \Gamma_{\alpha}^{\perp}, \gamma\right)=\left(S\left(\omega^{\prime}\right), h^{\prime}\left(\omega^{\prime}\right) \phi(g) \Gamma_{\alpha}^{\perp}, \gamma\left(\omega^{\prime}, g \Gamma_{\alpha}^{\perp}\right) \phi(\gamma)\right)
$$

for some $\gamma ; \Omega^{\prime} \times G / \Gamma_{\alpha}^{\perp} \rightarrow \Gamma_{\alpha}^{\perp}$.

As $\phi \mid \Gamma_{\bar{\alpha}}$ is ergodic, by Lind [6] the first two components split off with a Bernoulli complement. Furthermore, the entropy of $\phi$ on $G / \Gamma_{\alpha}^{\perp}$ is zero, and so the action on the first two components is of 0-entropy.

Proposition 3.6. Any $G$, $\phi$-extension $\hat{T}$ of a finite entropy Bernoulli shift $T$ is of the form Bernoulli $\times$ distal.

Proof. From our classification, we can write (without loss of generality)

$$
\hat{T}(\omega, g)=\left(T(\omega), h(\omega) g_{0} \phi(g)\right)
$$

where $h: \Omega \rightarrow H, g_{0} \phi(H) g_{0}^{-1}=H$, and

$$
\bar{T}(\omega, h)=\left(T(\omega), h(\omega) g_{0} \phi(h) g_{0}^{-1}\right)
$$

is a Bernoulli $g_{0}^{*} \circ \phi, H$-extension of $T$. Map $G$ to $H \backslash G \times H$, by the map $g \mapsto\left(H g, g \delta(H g)^{-1}\right)$, where $\delta: H \backslash G \rightarrow G$ is a cross section. Then $\hat{T}$ becomes isomorphic to

$$
\begin{aligned}
& \hat{T}^{\prime}(\omega, H g, h) \\
& =\left(T(\omega), H g_{0} \phi(g), h(\omega)\left(g_{0} \phi(h) g_{0}^{-1}\right)\left(g_{0} \phi(\delta(H g)) \delta\left(g_{0} \phi(H g)\right)^{-1}\right)\right) .
\end{aligned}
$$

Set $\Omega^{\prime}=H \backslash G, S(H g)=H g_{0} \phi(g)$,

$$
h^{\prime}(H g)=\left(g_{0} \phi(\delta(H g)) \delta\left(g_{0} \phi(H g)\right)^{-1}\right)^{-1} \text {. }
$$

Then

$$
\hat{T}^{\prime}\left(\omega, \omega^{\prime}, h\right)=\left(T(\omega), S\left(\omega^{\prime}\right), h(\omega)\left(g_{0} \phi(h) g_{0}^{-1}\right) h^{\prime-1}\left(\omega^{\prime}\right)\right) .
$$

Now for the algebraic trick. Write

$$
\hat{S}\left(\omega^{\prime}, h\right)=\left(S\left(\omega^{\prime}\right), h^{\prime}\left(\omega^{\prime}\right)\left(g_{0} \phi(h) g_{0}^{-1}\right)\right) \text {. }
$$

Now $S$ has entropy zero. Thus $\hat{S}$ is of the form Bernoulli $\times 0$-entropy, by the previous lemma. Hence $\bar{T} \times \hat{S}$ is Bernoulli $\times 0$-entropy, as $\bar{T}$ is Bernoulli. But the map

$$
\left(\omega, h_{1}\right)\left(\omega^{\prime}, h_{2}\right) \stackrel{\psi}{\mapsto}\left(\omega, \omega^{\prime}, h \circ h_{2}^{-1}\right)
$$

satisfies

$$
\psi(\bar{T} \times S)=\hat{T}^{\prime}(\psi),
$$

and $\hat{T}^{\prime}$ is a factor of $\bar{T} \times \hat{S}$. Then, by Thouvenout [12], $\hat{T}^{\prime}$ must be Bernoulli $\times 0$-entropy. 
Thus $\Pi(T)$ has a Bernoulli complement, and as $\hat{T} \mid \pi(\hat{T})$ is distal, we are done.

REMARK. The last result actually does not even require "freeness" of the $\mathbf{Z} \times{ }^{\phi} G$ action. Consider any action of $\mathbf{Z} \times{ }^{\phi} G$ whose $\mathbf{Z}$ part is free on the $G$-invariant factor. Then, in view of the remarks in the introduction, this action may be regarded as a factor of a free such action. Thus the $\mathbf{Z}$ part of our original action is a factor of a Bernoulli $\times$ distal action. Then it splits into Bernoulli $\times 0$-entropy, by [12]. Further, its Pinsker factor is a factor of the aforementioned distal action, and therefore is itself distal.

This now completes our classification of $G, \phi$-extensions of finite entropy Bernoulli shifts. The question still remains as to whether this classification also holds when $T$ has infinite entropy. There is little doubt that this is true, only one argument really being needed to obtain it. It needs to be shown that for any skewing function $\gamma(\omega)$ there is an $\alpha_{\omega}$ with

$$
\bar{\gamma}(\omega)=\alpha_{T(\omega)}^{-1} \gamma(\omega) \phi\left(\alpha_{\omega}\right)
$$

and $\bar{\gamma}$ is measurable with respect to a finite entropy factor of $T$. If this is true, standard arguments will lift the results of [8] and [9] to the infinite entropy case, and the rest of the argument will follow. Furthermore, using the structure of the action of $\hat{\phi}$, and Lind's work in [6], one really only need show (3.1) when $\phi$ is an isometry.

IV. Extending the classification to $\mathrm{Z} \times{ }^{\phi} G$ actions with conjugate isotropy subgroups. In the previous section we classified the free $\mathbf{Z} \times{ }^{\phi} G$ actions which, on the $\sigma$-algebra of $G$-invariant sets, are Bernoulli. This classification was in terms of the Pinsker algebra of the $\mathbf{Z}$ part of the action, which we saw was isomorphic to $g_{0} \phi$ acting on $H \backslash G$, for some subgroup $H$, and $g_{0}$ with $g_{0} \phi(H) g_{0}^{-1}=H$. There are many cases where freeness of the $\mathrm{Z} \times{ }^{\phi} G$ action cannot be guaranteed, but where one can guarantee that all the isotropy subgroups, a.s., are conjugate (see $\S \mathrm{V}$ ). What we will do now is show that for such "quasifree" actions we also have a classification. It is similar to that in the free case, and reduces to it, but is different enough to lead to some interesting structure.

Let $(\bar{T}, \tau)$ be a $\mathbf{Z} \times^{\phi} G$ action on $(\bar{\Omega}, \overline{\mathscr{F}}, \bar{\mu})$. For $\bar{\omega} \in \bar{\Omega}$, let $I_{\bar{\omega}}=\{g \in$ $\left.G \mid \tau_{g}(\bar{\omega})=\bar{\omega}\right\}$, the isotropy subgroup of $\omega$. Suppose for a.e. $\omega \in \bar{\Omega}, I_{\bar{\omega}}=$ $a_{\bar{\omega}} I a_{\omega}^{-1}$, where $I$ is some fixed closed subgroup of $G$ and $\bar{\omega} \mapsto a_{\bar{\omega}}$ is some $G$ valued function, which may in fact be chosen measurable. We will then say $(\bar{T}, \tau)$ is quasifree of type $I$.

Let $\overline{\mathbb{Q}} \subset \overline{\mathscr{F}}$ be the algebra of sets fixed by $\tau$. The fibers of $\overline{\mathcal{Q}}$ are the ergodic components of $\tau$. Let $T$ acting on $(\Omega, Q, \mu)$ be a copy of $\bar{T}$ acting on $\bar{Q}, p$ : $\bar{\Omega} \rightarrow \Omega$ the projection to the factor. The analysis of $\S I$ takes an especially simple form in this case: as the fibers $p^{-1}(\omega)$ are ergodic components of $\tau$, we may conclude that on a.e. $p^{-1}(\omega)$ there is a point $\beta(\omega)$ depending measurably on $\omega$ with $I_{\beta(\omega)}=I$. Thus the set $p^{-1}(\omega)$ can be identified as $I \backslash G$ with $\tau$ acting by right multiplication, and we can write $\bar{\Omega}=\Omega \times I \backslash G$. Now consider $\bar{T}(\omega, I)$, which must be $(T(\omega), I \gamma(\omega))$ for some $\gamma(\omega) \in b$. But as $\bar{T}$ and $\tau$ 
skew commute, for $i \in I$,

$$
\begin{aligned}
\bar{T} \tau_{i}(\omega, I) & =\bar{T}(\omega, I)=(T(\omega), I \gamma(\omega)) \\
& =\tau_{\phi(i)} \bar{T}(\omega, I)=(T(\omega), I \gamma(\omega) \phi(i)),
\end{aligned}
$$

and so $I \gamma(\omega)=\gamma(\omega) \phi(I)$, or

$$
\gamma(\omega)^{-1} I \gamma(\omega)=\phi(I) .
$$

Now (4.1) is a very strong restriction; it says $\phi(I)$ and $I$ are conjugate, and furthermore $\gamma(\omega)$, for all $\omega$, conjugates $I$ to $\phi(I)$. Hence we must have

$$
\phi(I)=\bar{g}^{-1} I \bar{g} \quad \text { for some } \bar{g} \in G,
$$

and $\gamma: \Omega \rightarrow N_{G}(I) \bar{g}$, where $N_{G}(I)$ is the normalizer in $G$ of $I$, so $\gamma(\omega)=\nu(\omega) \bar{g}$, $\nu: \Omega \rightarrow N_{G}(I)$. Thus for any quasifree $\mathbf{Z} \times{ }^{\phi} G$ action of type $I$ to exist we must have $\bar{g} \phi(I) \bar{g}^{-1}=I$, for some $\bar{g}$, and then the $\mathbf{Z}$ part of the action can be written as

$$
\bar{T}(\omega, I g)=(T(\omega), I \nu(\omega) \bar{g} \phi(g))
$$

where $\nu: \Omega \rightarrow N_{G}(I)$.

Now we classify actions, up to isomorphism, of the form (4.2) where $T$ is a Bernoulli shift of entropy $a<\infty$.

Suppose we have two actions $\bar{T}(\omega, I g)=(T(\omega), I \nu(\omega) \bar{g} \phi(g))$ and $\bar{T}^{\prime}(\omega, I g)$ $=\left(T(\omega), I \nu^{\prime}(\omega) \bar{g} \phi(g)\right)$. What would it mean for the corresponding $\mathbf{Z} \times{ }^{\phi} G$ actions to be isomorphic? Let $\bar{\psi}$ be the isomorphism. Now $\bar{\psi}$ must take the $G$-invariant algebras of sets to each other, so $\bar{\psi}(\omega, I)=(\psi(\omega), I \alpha(\omega))$, hence $\bar{\psi}(\omega, I g)=(\psi(\omega), I \alpha(\omega) g)$, and if $g \in I$ we get $I \alpha(\omega)=\alpha(\omega) I$, so $\alpha: \Omega \rightarrow$ $N_{G}(I)$. Then

$$
\bar{\psi}(\omega, I g)=(\psi(\omega), \alpha(\omega) I g),
$$

and since $\bar{\psi} \bar{T}=\bar{T}^{\prime} \bar{\psi}$, we get

$$
I \alpha(T(\omega)) \nu(\omega)=I \nu^{\prime}(\omega) \bar{g} \phi(\alpha(\omega)) \bar{g}^{-1} .
$$

If we replace the actions $(T, \tau)$ and $\left(T^{\prime}, \tau^{\prime}\right)$ by $\left(\tau_{\bar{g}} T, \tau\right)$ and $\left(\tau_{\bar{g}}^{\prime} T^{\prime}, \tau^{\prime}\right)$, we have rewritten the $\mathbf{Z} \times^{\phi} G$ actions as $\mathbf{Z} \times{ }^{\psi} G$ actions, where $\psi=\bar{g}^{*} \circ \phi$; recall that $\bar{g}^{*}$ means conjugation by $\bar{g}$. Since $\bar{g} \phi(I) \bar{g}^{-1}=I$, we also have

$$
\bar{g} \phi\left(N_{G}(I)\right) \bar{g}^{-1}=N_{G}(I),
$$

so $\tau_{\bar{g}} T$ and $\tau_{\bar{g}}^{\prime} T^{\prime}$ leave $\Omega \times\left(I \backslash N_{G}(I)\right)$ invariant, and (4.3) says the two original $\mathbf{Z} \times{ }^{\phi} G$ actions are isomorphic iff the two $\mathbf{Z} \times{ }^{\psi}\left(I \backslash N_{G}(I)\right)$ actions $\left(\tau_{\bar{g}} T\left|\Omega \times\left(I \backslash N_{G}(I)\right), \tau\right| \Omega \times\left(I \backslash N_{G}(I)\right)\right)$ and $\left(\tau_{\bar{g}}^{\prime} T^{\prime}\left|\Omega \times\left(I \backslash N_{G}(I)\right), \tau^{\prime}\right| \Omega \times\right.$ $\left.\left(I \backslash N_{G}(I)\right)\right)$ are isomorphic. The latter are free, and hence have been classified by the previous section. Let $\mathcal{K}_{I}(\bar{T})$ be $\mathcal{H}\left(\tau_{\bar{g}} \bar{T} \mid \Omega \times\left(I \backslash N_{G}(I)\right)\right)$, the classification map for free $Z \times{ }^{\psi}\left(I \backslash N_{G}(I)\right)$ actions. Thus we now have the following.

Proposition 4.1. Two quasifree $\mathrm{Z} \times{ }^{\phi} G$ actions $(\bar{T}, \tau)$ and $\left(\bar{T}^{\prime}, \tau^{\prime}\right)$ of type $\mathrm{I}$ which when restricted to their algebras of $G$-invariant sets are Bernoulli of entropy $a<\infty$ are isomorphic iff $\mathcal{H}_{I}(\bar{T})=\mathcal{H}_{I}(\bar{T})$.

Notice that if $I=\{$ id $\}$ then $N_{G}(I)=G$, and $\bar{g}=\mathrm{id}$, so this reduces to the 
earlier classification. Unlike the free case, the classification of Proposition 4.1 is not given in terms of $T \mid \Pi(\bar{T})$, but rather in terms of the Pinsker algebra of $\tau_{\bar{g}} \bar{T}$ restricted to an invariant subset, perhaps of measure zero. What we will do now is to characterize the action of $\bar{T}$ on its Pinsker algebra, seeing how it relates to the Pinsker algebra of $\tau_{g^{-1}} \bar{T}$ on $\Omega \times\left(I \backslash N_{G}(I)\right)$.

Let

$$
\mathcal{H}_{I}(\bar{T})=\left\langle I \backslash \tilde{H} I, \tilde{g}_{0} I\right\rangle, \tilde{H} I \subset N_{G}(I),
$$

$\tilde{H}$ containing the maximal subgroup $\tilde{\Gamma}$ of $I \backslash N_{G}(I)$ on which $\bar{g}^{*} \phi$ is ergodic (Bernoulli), and

$$
\tilde{g}_{0} \bar{g}(\tilde{H} I) \bar{g}^{-1} \tilde{g}_{0}^{-1}=\tilde{H} I .
$$

We can modify $\nu(\omega)$ by a coboundary to be $\eta(\omega) \tilde{g}_{0}$ where $\eta: \Omega \rightarrow \tilde{H} I$. Hence we can rewrite $\bar{T}$ as

$$
T(\omega, I g)=\left(T(\omega), I \eta(\omega) \bar{g}^{\prime} \phi(g)\right)
$$

where $\bar{g}^{\prime}=\tilde{g}_{0} \bar{g}$, and $\eta: \Omega \rightarrow \tilde{H} I$.

Now notice that

$$
\bar{T}\left(\left(\Omega,\left(H I \Gamma_{\bar{\alpha}}\right) g\right)\right)=\left(\Omega,\left(\tilde{H} I \Gamma_{\bar{\alpha}}\right) \bar{g}^{\prime} \phi(g)\right) .
$$

Since $\tilde{H} I \Gamma_{\alpha}^{\perp} \supset \Gamma_{\alpha}^{\perp}, \bar{T}$ acting on fibers $\left(\Omega, \tilde{H} I \Gamma_{\alpha}^{\perp}\right)$ has entropy 0 , and so this factor of $\bar{T}$ is in $\Pi(\bar{T})$. We want to show that it is in fact all of $\Pi(\bar{T})$.

Let $\hat{T}(\omega, g)=\left(T(\omega), \eta(\omega) \bar{g}^{\prime} \phi(g)\right)$, a free $\mathbf{Z} \times^{\phi} G$ action. Now $\bar{T}$ is a factor of this, so $\Pi(\bar{T})$ is $\Pi(\hat{T})$ restricted to this factor. Call such a lifting $\hat{T}$ of $\bar{T}$ a "full action" of $\bar{T}$. It is not unique, even up to isomorphism. Let $\mathcal{H}(\hat{T})=$ $\left\langle H, g_{0}\right\rangle$. Thus an atom of $\Pi(\hat{T})$ has the form $\cup_{\omega \in \Omega}(\omega, \alpha(\omega) H g)$, for some cross section $\alpha(\omega)$. From the previous paragraph we know that sets of the form $\left(\Omega, \tilde{H} I \Gamma_{\alpha}^{\perp} g^{\prime}\right)$ must be unions of such fibers. Suppose for some $g, g^{\prime}$, and a.e. $\omega$,

$$
\alpha(\omega) H g \subset \tilde{H} I \Gamma_{a}^{\perp} g^{\prime} .
$$

Replace $H$ by $H^{\prime}=g^{\prime} g^{-1} H g g^{\prime-1}$, and $\alpha(\omega)$ by $\alpha^{\prime}(\omega)=\alpha(\omega) g g^{\prime-1}$. Now $\alpha^{\prime}(\omega) H^{\prime}$ $\subset \tilde{H} I \Gamma_{\alpha}^{\perp}$ for a.e. $\omega$. Pick an $h \in H^{\prime}$, so that for some $\omega_{0}, \alpha^{\prime}\left(\omega_{0}\right) h \in \tilde{H} I \Gamma_{\bar{\alpha}}$. Let $\alpha^{\prime \prime}(\omega)=\alpha^{\prime}(\omega) h$. Now $\alpha^{\prime \prime}(\omega) H^{\prime} \subset \tilde{H} I \Gamma_{a}^{\perp}$ and $\alpha^{\prime \prime}\left(\omega_{0}\right) \in H I \Gamma_{\alpha}^{\perp}$, hence $H^{\prime}$ $\subset \tilde{H} I \Gamma_{\alpha}^{\perp}$ and $\alpha^{\prime \prime}: \Omega \rightarrow H I \Gamma_{\alpha}^{\perp}$. Now the atoms of $\Pi(\bar{T})$ are written

$$
\bigcup_{\omega \in \Omega}\left(\omega, \alpha^{\prime \prime}(\omega) H^{\prime} g\right), \quad \alpha^{\prime \prime}: \Omega \rightarrow H I \Gamma_{\bar{\alpha}}^{\perp} .
$$

But now $\Gamma_{\alpha}^{\perp} \subset H^{\prime}$ from Lemma 3.3, hence we can assume $\alpha^{\prime \prime}: \Omega \rightarrow \tilde{H} I \subset$ $N_{G}(I)$. Thus replacing $\eta(\omega) \bar{g}^{\prime} \phi\left(\alpha^{\prime \prime}(\omega)\right)$, we know

$$
T^{\prime}(\omega, g)=\left(T(\omega), \alpha^{\prime \prime}(\omega) \phi(g)\right)
$$

is $G, \phi$-isomorphic to $\hat{T}$, but furthermore the isomorphism (id, $\alpha^{\prime \prime}$ ) takes the factor $\Omega \times I \backslash G$ to itself. Finally, we have unwound the Pinsker algebra, and so $\gamma^{\prime \prime}(\omega)=\eta^{\prime \prime}(\omega) g_{0}^{\prime \prime}, \eta^{\prime \prime}: \Omega \rightarrow H^{\prime \prime}, g_{0}^{\prime \prime} \phi\left(H^{\prime \prime}\right) g_{0}^{\prime \prime-1}=H^{\prime \prime}$.

Now as $\bar{g}^{\prime} \phi(\tilde{H} I) \bar{g}^{\prime-1}=\tilde{H} I$, from $(4.4) g^{\prime \prime}(\omega) \bar{g}^{-1} \in \tilde{H} I$, hence

$$
\eta^{\prime \prime}(\omega) g_{0}^{\prime \prime}=\tilde{\eta}(\omega) \bar{g}, \text { for some } \tilde{\eta}: \Omega \rightarrow \tilde{H} I \text {. }
$$


Now $g_{0}^{\prime \prime}$ is only unique up to its $H^{\prime \prime}$ coset, and similarly $\bar{g}$ up to its $\tilde{H} I$ coset. For some $\omega_{0}$ replace $g_{0}^{\prime \prime}$ by $\eta^{\prime \prime}\left(\omega_{0}\right) g_{0}^{\prime \prime}$ and $\bar{g}$ by $\tilde{\eta}\left(\omega_{0}\right) \bar{g}$. Hence w.l.o.g. We may assume $\eta^{\prime \prime}(\omega)=\tilde{\eta}(\omega) \subset H^{\prime \prime} \cap \tilde{H} I$ and $g_{0}^{\prime \prime}=\bar{g}$. Set $H^{\prime \prime} \cap \tilde{H} I=\tilde{H}^{\prime}$.

Now $\Gamma_{a}^{\perp} \subset H^{\prime \prime}$ and $\tilde{\Gamma} \subset \tilde{H} I \backslash I$, but furthermore as $\bar{g}^{*} \circ \phi$ acting on $N_{G}(I)_{\Gamma_{\alpha}^{\perp} I}$ is distal, $\tilde{\Gamma} I \subset \Gamma_{\alpha}^{\perp} I$. Hence $\tilde{\Gamma} I \subset H I$ and our latest representation of $\bar{T}$, where

$$
\bar{T}(\omega, \operatorname{In})=\left(T(\omega), \eta^{\prime \prime}(\omega) \bar{g} \phi(n)\right),
$$

on the factor $\{\phi, \Omega\} \times\left(\tilde{H}^{\prime} I \backslash N_{G}(I)\right)$, has entropy 0 . We already know $\tilde{H}^{\prime} I \subset \tilde{H} I$. But $\left(\Omega, \tilde{H} I g^{\prime}\right)$ are the atoms of $\Pi(\bar{T})$, hence $\tilde{H}^{\prime} I \supset \tilde{H} I$ and so $\tilde{H}^{\prime} I=H^{\prime \prime} \cap \tilde{H} I=\tilde{H} I$. Hence $\tilde{H} I \subset H^{\prime \prime} I$ and so $\tilde{H} I \Gamma_{a}^{\perp} \subset H^{\prime \prime} I$.

We already know $\tilde{H} I \Gamma_{\alpha}^{\perp} \supset H^{\prime \prime} I$, so $H^{\prime \prime} I=\tilde{H} I \Gamma_{\alpha}^{\perp}$. This gives the following.

Proposition 4.2. Let $(\bar{T}, \tau)$ be a quasifree $\mathrm{Z} \times{ }^{\phi} G$ action of type $\mathrm{I}$ with $\mathcal{H}_{I}(\bar{T})=\left\langle\tilde{H}, I \tilde{g}_{0}\right\rangle$. If $\hat{T}$ is any full action of $\bar{T}$, and $\mathcal{H}(\hat{T})=\left\langle H, g_{0}\right\rangle$, then the representatives $\left(\tilde{H}, I \tilde{g}_{0}\right)$ and $\left(H, g_{0}\right)$ can be chosen so that $\tilde{g}_{0}=g_{0}$ and $\tilde{H} I \Gamma_{a}^{\perp}=$ HI.

This result has some interesting corollaries.

COROllary 4.3. Let $\overline{\mathcal{H}}_{I}(\bar{T})=\left\langle H I, I g_{0}\right\rangle$ where for some full action $\hat{T}$ of $\bar{T}$, $\mathcal{H}(\hat{T})=\left\langle H, g_{0}\right\rangle$. Then $\overline{\mathcal{H}}_{I}(T)$ is an isomorphism invariant of the quasifree action, and is a complete invariant iff $\tilde{\Gamma}=\Gamma_{\alpha}^{\perp} \cap N_{G}(I)$.

Proof. It is obvious from Proposition 4.2 that $\mathcal{C}(\bar{T})$ is an invariant. If $\tilde{\Gamma}=\Gamma_{a}^{\perp} \cap N_{G}(I)$ then

$$
\mathcal{K}_{l}(\bar{T})=\left\langle H I \cap N_{G}(I), I g_{0}\right\rangle,
$$

as $\tilde{H} I=\tilde{H} I \Gamma_{\bar{\alpha}} \cap N_{G}(I)$ since $\tilde{\Gamma} \subset \tilde{H}$. If $\tilde{\Gamma} \subset \Gamma_{\bar{\alpha}}^{\perp} \cap N_{G}(I)$, let $(\bar{T}, \tau)$ and $\left(\bar{T}, \tau^{\prime}\right)$ have

$$
\mathcal{K}_{I}(\bar{T})=\langle\tilde{\Gamma} I, I\rangle, \quad \mathcal{K}_{I}\left(\bar{T}^{\prime}\right)=\left\langle I\left(\Gamma_{\bar{\alpha}} \cap N_{G}(I)\right), I\right\rangle .
$$

Now $(\bar{T}, \tau)$ and $\left(\bar{T}^{\prime}, \tau\right)$ are not isomorphic, but

$$
\overline{\mathscr{K}}_{I}(\bar{T})=\left\langle\tilde{\Gamma} I \Gamma_{\alpha}^{\perp}, I\right\rangle=\left\langle\left(\Gamma_{\alpha}^{\perp} \cap N_{G}(I)\right) I \Gamma_{\alpha}^{\perp}, I\right\rangle=\mathcal{K}_{I}\left(\bar{T}^{\prime}\right) .
$$

This noncompleteness of the invariant $\overline{\mathcal{H}}_{I}$ actually does arise, as we shall see in $\S \mathrm{VI}$, and gives rise to nonisomorphic quasifree actions whose $\mathbf{Z}$ parts are Bernoulli and of equal entropy. Here is a simple and interesting corollary of Proposition 4.2.

Corollary 4.4. A quasifree $\mathrm{Z} \times{ }^{\phi} G$ action of type $\mathrm{I}$ whose $\mathrm{Z}$ part is Bernoulli exists iff $N_{G}(I) \Gamma_{a}^{\perp}=G$.

Proof. If $N_{G}(I) \Gamma_{a}^{\perp} \neq G$, then $\overline{\mathcal{H}}_{I}(\bar{T})=\left\langle H I, I g_{0}\right\rangle$ must have $H I \subset$ $N_{G}(I) \Gamma_{\bar{\alpha}} \neq G$. Hence $\Pi(\bar{T})$ is not trivial and $\bar{T}$ is not even $K$. But if $N_{G}(I) \Gamma_{\alpha}^{\perp}=G$, let $\bar{T}$ have $\mathcal{H}_{I}(\bar{T})=\left\langle N_{G}(I), I\right\rangle$, and then $\overline{\mathcal{K}}_{I}(\bar{T})=\langle G, I\rangle$ so $\Pi(\bar{T})$ is trivial, and by Proposition 3.6, $\bar{T}$ is Bernoulli. 
ExAMPLE. If $I$ is not a normal subgroup of $G$, and $\phi$ is the identity automorphism, then $\Gamma_{\alpha}^{\perp}=\{$ id $\}$, so $N_{G}(I) \Gamma_{\alpha}^{\perp}=N_{G}(I) \neq G$, and therefore no action of $\mathbf{Z} \times{ }^{\phi} G$ which is quasifree of type I can have Bernoulli $\mathbf{Z}$ part.

However, for connected Lie groups a much more striking phenomenon will be seen in the next section: namely, necessary and sufficient that an action of $\mathbf{Z} \times{ }^{\phi} G$ exist with Bernoulli $\mathbf{Z}$ part is that $I$ be normal and $\phi$-invariant!

\section{The case when $G$ is a Lie group.}

Proposition 5.1. If $G$ is a Lie group, then for any automorphism $\phi$ and any action $(\bar{T}, \tau)$ of $\mathrm{Z} \times{ }^{\phi} G$ for which the restriction $T$ of $\bar{T}$ to the $\sigma$-field of invariant sets of $\tau$ is weak mixing, $\tau$ must be quasifree.

Proof. Consider the set $\mathscr{K}$ of closed subgroups of $G$ as a subset of all closed subsets of $G$. It inherits a natural topology (cf. [2]) in which it is a standard Borel space. Moreover, $G$ acts on $\mathscr{K}$ by conjugation to give a Borel $G$-space. Let $\mathcal{K}$ be the quotient space of $\mathscr{K}$ by the action of $G$, i.e. the set of conjugacy classes of subgroups. Since $G$ is compact, $\hat{K}$ is a standard Borel space in the inherited Borel structure.

Now consider the map $\omega \mapsto H_{\omega}$ given in the analysis of $\bar{T}$ in $\S \mathrm{I}$. This may be taken to be a Borel map. Furthermore, $H_{T \omega}=\phi\left(H_{\omega}\right)$. Use the map $\omega \mapsto H_{\omega}$ to transfer the measure $\mu$ on the space of $T$ to $\hat{\kappa} \hat{\kappa}$; let $\hat{\mu}$ be the image measure. Then the measure $\hat{\mu}$ on $\hat{\mathcal{K}}$, together with the map $\hat{H} \rightarrow \hat{\phi}(\hat{H})=$ $\phi(H)$ gives a finite measure-preserving transformation, which is a factor of $T$ because of the relation $H_{T \omega}=\hat{\phi}\left(\hat{H}_{\omega}\right)$. Since $T$ is weakly mixing, $\hat{\phi}$ must be either strictly ergodic, or the trivial transformation on a space of one point. We shall show that the latter holds, which will prove our proposition.

This will be done by showing that $\mathcal{K}$ is in fact countable. This has the status of a folklemma; we indicate below why it holds.

The countability of $\hat{K}$ is an immediate corollary of a theorem of Montgomery and Zippin [7] about compact subgroups of Lie groups. More specifically: if $G$ is any Lie group, give $\mathcal{K}$ the topology it inherits as a subset of the set of all closed subsets $\mathcal{S}(\bar{G})$ of the one point compactification of $G$. This is compact, and will be metrizable if $G$ is second countable. Let $\mathcal{K}_{n}$ be those $H \in \mathcal{K}$ of dimension $n$. Then $\mathcal{S}(\bar{G})$ is a topological $G$-space for the action of $G$ by conjugation and so are $\mathscr{K}$ and the sets $\mathcal{K}_{n}$. The following we believe to be well known, and follows quickly from [7].

Proposition 5.2. Every G-orbit of a point $H$ of $\mathcal{K}_{n}$ is open in $\mathcal{K}_{n}$, and this orbit is homeomorphic to the coset space $G / N(H)$ where $N(H)$ is the normalizer of $H$. Hence if $G$ is second countable, so that $\mathcal{K}_{n}$ is a separable metric space, there can only be a countable number of $G$-orbits in $\mathscr{K}_{n}$ for each $n$, and hence there can only be a countable number of $G$ orbits in $\mathcal{K}$.

We combine the above with some special features of connected Lie groups to obtain the following results. 
THEOREM 5.3. Let $G$ be a compact connected Lie group, $\phi$ an automorphism of $G$. Then:

(a) for every $\phi$-invariant normal subgroup $I$ of $G$ and every $a>0$ there exists (up to isomorphism) precisely one action $(\bar{T}, \tau)$ of $\mathbf{Z} \times{ }^{\phi} G$ with $\bar{T}$ Bernoulli of entropy $a+h\left(\phi^{\prime}\right)$, where $\phi^{\prime}$ is the action of $\phi$ on $G / I$, with isotropy groups all equal to I;

(b) there exist no other actions of $\mathbf{Z} \times^{\phi} G$ whose $\mathbf{Z}$ part is Bernoulli.

Proof. (a) is obvious from the results of [9] and §II for the free case, applied to $\mathbf{Z} \times \phi^{\phi^{\prime}}(G / I)$. As for (b): assume we are given an action $(\bar{T}, \tau)$ of $\mathbf{Z} \times{ }^{\phi} G$ with $\bar{T}$ Bernoulli.

By Proposition 5.1 we know that the action is quasifree with almost all isotropy groups lying in some $\phi$-invariant conjugacy class $I$ of subgroups of $G$. Let $Z_{0}$ be the connected component of the center of $G$, and let $R$ be any irreducible representation of $G$ which is trivial on $Z_{0}$. Then $R$ is essentially a representation of the connected semi-simple group $G / Z_{0}$. The automorphism $\phi$ leaves $Z_{0}$ invariant and hence induces an automorphism $\phi_{0}$ of $G / Z_{0}$. By standard results, some power of $\phi_{0}$ is inner and therefore the orbit of $R$ under $\phi_{0}$ and hence the orbit of $R$ under $\phi$ is finite. Since the kernels of such representations intersect in $Z_{0}$, it follows that the subgroup $\Gamma_{\alpha}^{\perp}$ of $G$ must be contained in $Z_{0}$. Now $Z_{0}$ normalizes any subgroup $I$, so $N_{G}(I) \supset Z_{0} \supset \Gamma_{\alpha}^{\perp}$. Thus the necessary condition in Proposition 4.2 for existence of a $Z \times{ }^{\phi} G$ action with Bernoulli $\mathbf{Z}$ part and quasifree with isotropy groups in $I$ implies here that $N_{G}(I)=G$ so that $I$ is normal, and hence also invariant under $\phi$.

VI. Some examples. These will all be counterexamples to the "isomorphism theorem" for actions of $\mathbf{Z} \times{ }^{\phi} G$ which are Bernoulli on $\mathbf{Z}$. More precisely: in each case there will be a given action of $G$ and a given Bernoulli transformation $T$ on the invariant $\sigma$-algebra of $\tau$. In each case there will be different ways of lifting $T$ to a transformation on the space where $\tau$ acts, so that the lifted transformations skew-commute properly with $\tau$, and are Bernoulli shifts of equal entropy, but for which the corresponding $\mathbf{Z} \times{ }^{\phi} G$ actions are not isomorphic.

Example 1. $G$ will be a disconnected (two component) Lie group. Let $\mathbf{T}$ be a torus of any dimension and let $\theta$ be the automorphism which sends $x$ to $x^{-1}$. We let $G$ be the semi-direct product of $T$ with the two element group $Z_{2}$, with $Z_{2}$ acting by $\theta$, and let $\sigma$ denote the nontrivial element of $Z_{2}$. Now let $\phi_{1}$ be any ergodic automorphism of $T$ and extend $\phi_{1}$ to an automorphism $\phi$ of $G$ by $\phi\left(t, \sigma^{e}\right)=\left(\phi_{1}(t), \sigma^{e}\right)$ with $\varepsilon=0,1, t \in \mathbf{T}$. This is indeed an automorphism of $G$ since $\theta$ and $\phi$ commute.

We choose the subgroup $I$ to be the two element group $Z_{2}$ generated by $\sigma$. A simple calculation shows that the normalizer $N_{G}(I)$ is the (semi)direct product of the elements of order two in $\mathbf{T}$, call them $\mathbf{T}_{2}$, with $\mathbf{Z}_{2}$; it is an elementary abelian 2-group of order $2^{\operatorname{dim}(T)+1}$. It is easy to see that $\Gamma_{\alpha}^{\perp}$, the 
maximal subgroup of $G$ on which $\phi$ is ergodic, is $T$, the connected component of $G$. But clearly the corresponding subgroup $\tilde{\Gamma}$ of $N_{G}(I)$ is trivial. Hence $\tilde{\Gamma} \neq \Gamma_{\alpha}^{\perp} \cap N_{G}(I)$.

The classification scheme for quasifree $\mathbf{Z} \times{ }^{\phi} G$ actions of type I given by Propositions 4.1 and 3.4 can be explicitly calculated; note that $N_{G}(I) / I \simeq \mathbf{T}_{2}$ is a vector space of dimension $n=\operatorname{dim} \mathbf{T}$ over the two element field $Z_{2}$ and that $\phi$ gives rise to an invertible linear transformation of $T_{2}$ to itself. If we pick a $\phi$ invariant subspace $H$ of $\mathrm{T}_{2}$ and a vector $v$ in the quotient $\mathrm{T}_{2} / H$, then such a pair $(H, v)$, the Pinsker invariant, completely parameterizes the quasifree $\mathbf{Z} \times{ }^{\phi} G$ actions with isotropy $\hat{I}$.

On the other hand, since already $I \cdot G_{1}=G$, by Proposition 4.2 all such actions have Bernoulli $\mathbf{Z}$ part. Thus we have nonuniqueness in this case of a (disconnected) Lie group, but with nevertheless a firm hold on the amount of nonuniqueness.

EXAMPLE 2. $G$ connected but not Lie, and $\tau$ quasifree.

Let $G_{0}$ be a simple, connected Lie group, let $G=G_{0}^{\mathbf{Z}}$, and let $\phi$ be the shift on $G$. Now let $I_{0}$ be a maximal torus in $G_{0}$, with Weyl group $W=N_{G_{0}}\left(I_{0}\right) / I_{0}$. Let $I$ be the diagonally embedded copy of $I_{0}$ in $G$. $I$ is clearly $\phi$-invariant. The normalizer of $I$ is readily seen to contain $I_{\infty}$, the infinite product of copies of $I_{0}$, and $N_{G}(I) / I_{\infty}$ is also readily seen to be isomorphic to the finite group $W$. Now since $\phi$ is already Bernoulli on both $G$ and $I$, the classification theorems for quasifree $\mathbf{Z} \times{ }^{\phi} G$ actions of type I show that they are automatically Bernoulli if the action on the $G$ invariants algebra of sets is Bernoulli, and that they are classified by $\mathrm{Z} \times{ }^{\phi}\left(N_{G}(I) / I\right)$ actions; the latter are then classified by their Pinsker invariant which in this case amounts to a pair $(H, \dot{g})$ where $H$ is a (conjugacy class of) subgroups of the Weyl group $W$ and $\dot{\boldsymbol{g}}$ is an element of $W / H$. Only one of these, namely the one with $H=W$, corresponds to a Bernoulli $\mathrm{Z} \times{ }^{\phi}\left(N_{G}(I) / I\right)$ action. In this case, the number of classes of $\mathbf{Z} \times{ }^{\phi} G$ Bernoulli actions with isotropy groups specified to be in $\hat{I}$ is again finite with more than one element, and is parameterized quite explicitly.

EXAmple 3. $G$ abelian, but $\tau$ not quasifree. We are going to build a skew product over a Bernoulli base $(T, \Omega)$ where the fiber over $\omega$ is $G / H, H_{\omega}$ a closed subgroup of $G$. The proof of the isomorphism theorem for free actions rests heavily on the possibility of extending automorphisms of the base action to automorphisms of $(\hat{T}, \tau)$. If, in our example, $H_{\omega}$ were unique to $\omega$, then the only possible automorphisms of the action to itself would be the identity on the base factor, making its Bernoullicity of no value. Here is an example that has these properties.

Set $\bar{G}=\left(\mathbf{Z}_{2}\right)^{\mathrm{Z}}, \bar{\phi}$ the shift on $G$. Let $(T, \Omega)$ be the two shift, i.e. another copy of $(\phi, G)$. Set $H$ to be the two element subgroup $\{\omega$, id $\} G$. Let $X=$ $\cup_{\omega \in \Omega}\left(\omega \times \bar{G} / H_{\omega}\right)$ with measure $\mu \times \nu$ as a factor of $\Omega \times G$. Let $\hat{T}\left(\omega, g H_{\omega}\right)$ $=\left(T(\omega), \bar{\phi}(g) H_{T(\omega)}\right)$. This is Bernoulli, as it is a factor of $T \times \bar{\phi}$. Let $(\hat{T}, \tau)$ be the action of $\mathbf{Z} \times{ }^{\bar{\phi}} \bar{G}$. Any automorphism of $(T, \tau)$ must be of the form 
(id, $\alpha(\omega)$ ); for any point $\left(\omega, g H_{\omega}\right)$ is fixed by $\tau_{h}, h \in H_{\omega}$, hence must map to such a point. Even though this action is not free, it has the weaker property that the set of points fixed by any particular nonidentity element of $\mathbf{Z} \times{ }^{\bar{\phi}} \bar{G}$ has measure zero.

Now for our two examples. Set $G=\bar{G} \times \mathbf{Z}_{3}, \phi=\bar{\phi}$ id. Define

$$
\gamma(\omega)=\omega_{0}, \quad \gamma^{\prime}(\omega)=-\omega_{0},
$$

as elements of $\mathbf{Z}_{3}$. Now set

$$
\begin{aligned}
\hat{T}_{1}(\omega, \bar{g} H, i) & =\left(T(\omega), \bar{\phi}(\bar{g}) H_{T(\omega)}, \gamma(\omega) \circ i\right), \\
\hat{T}_{2}\left(\omega, \bar{g} H_{\omega}, i\right) & =\left(T(\omega), \bar{\phi}(\bar{g}) H_{T(\omega)}, \gamma^{\prime}(\omega) \circ i\right) .
\end{aligned}
$$

Then $\hat{T}_{1}$ and $\hat{T}_{2}$ are three point extensions of $\hat{T}$, and it is an easy check that they are Bernoulli, and they obviously have equal entropy. They give the $Z$ parts of two $\mathbf{Z} \times{ }^{\phi} G$ actions.

These two actions cannot be isomorphic. For any isomorphism must have the form (id, $\alpha(\omega), \beta(\omega)$ ), $\alpha: \Omega \rightarrow \bar{G}, \beta: \Omega \rightarrow \mathbf{Z}_{3}$ and

hence

$$
\gamma(\omega)=\beta(T(\omega)) \gamma^{\prime}(\omega) \beta^{-1}(\omega),
$$

$$
\beta(T(\omega)) \beta^{-1}(\omega)=\gamma(\omega) \gamma^{\prime}(\omega)^{-1}=\gamma^{\prime}(\omega),
$$

so $\gamma^{\prime}$ is a coboundary of $T$, and therefore may be replaced by the identity in $T_{2}$. But then $T_{2}$ would be nonergodic. On the other hand the map $\psi$ : $(\omega, \bar{g}, i) \mapsto(\omega, \bar{g},-i)$ is an isomorphism between $\hat{T}_{1}$ and $\hat{T}_{2}$ preserving the base factor.

\section{REFERENCES}

1. L. Auslander and C. C. Moore, Unitary representations of solvable Lie groups, Mem. Amer. Math. Soc., no. 62, 1966.

2. J. M. G. Fell, A Hausdorff topology for the closed subsets of a locally compact non-Hausdorff space, Proc. Amer. Math. Soc. 13 (1962), $472-476$.

3. H. Furstenburg, The structure of distal flows, Amer. J. Math. 85 (1963), 477-515.

4. E. Hewitt and K. A. Ross, Abstract harmonic analysis. II, Grundlagen der Math. Wissenchaften in Einzeldarstellungen, Band 152, Springer-Verlag, Berlin, 1970.

5. D. Lind, The structure of skew products with group automorphisms, Israel J. Math. (to appear).

6. Split skew products, a related functional equation, and specification, preprint, Univ. of Washington, 1977.

7. D. Montgomery and L. Zippin, A theorem on Lie groups, Bull. Amer. Math. Soc. 48 (1942), 448-452.

8. D. J. Rudolph, Classifying the isometric extensions of a Bernoulli shift, Israel J. Math. (to appear).

9. , An isomorphism theory for Bernoulli free Z-skew-compact group actions, Advances in Math. (to appear).

10. J. P. Thouvenot, Quelques propriétés des systèmes dynamiques qui se décompasent en un produit de deux systèmes dont l'un est un schéma de Bernoulli, Israel J. Math. 21 (1975), 177-207. 
11. __ Remarques sur les systèmes dynamiques donnes avec plusiers facteurs, Israel J. Math. 21 (1975), 215-232.

12. Une classe de systèmes pour lesquels la conjecture de Pinsker est oraie, Israel J. Math. 21 (1975), 208-214.

Departmant of Mathematics, University of Calufornia, Bbrkbley, California 94720 (Current address of J. Feldman and C. C. Moore)

Departmient of Mathimatics, Stanford University, Stanford, California 94350 (Current address of D. J. Rudolph) 